\title{
Out-of-step oscillation splitting criterion based on bus voltage frequency
}

\author{
Fei TANG, Jian YANG, Qingfen LIAO (ه), \\ Yifei WANG, Jun JIA
}

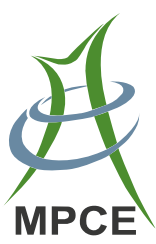

\begin{abstract}
Under the background of complicated interconnected network, the splitting criterion for accurately capturing the electrical center in real time is the prerequisite of power grid splitting. This paper studies the features of electric quantity in the electrical center in aspect of the instantaneous frequency, and proposes the out-of-step splitting criterion for power systems based on bus voltage frequency. Firstly, through the establishment and solution to the out-of-step model of the power grid, the analytical expression of the voltage frequency at any position is obtained in the out-of-step oscillation, and the voltage frequency features of electrical center and non-electrical center are analyzed in details. Then, this paper constructs the typical scene of migration of electrical center to study the change rules of voltage frequency. Finally, the splitting criterion based on bus voltage frequency is proposed as well as the instruction for use. This criterion is easy to be realized and can adapt to the migration of electrical center. Also it is free from the limits of power network structure and operational mode. Simulation results of CEPRI-36 system and interconnected network example of one actual region verify the accuracy and the effectiveness of the proposed criterion.
\end{abstract}

Keywords Out-of-step splitting, Voltage frequency, Electrical center, Splitting criterion

CrossCheck date: 2 April 2015

Received: 16 September 2014 / Accepted: 22 April 2015/Published online: 19 July 2015

(C) The Author(s) 2015. This article is published with open access at Springerlink.com

F. TANG, Q. LIAO, Y. WANG, J. JIA, School of Electrical

Engineering, Wuhan University, Wuhan 430072, China

$(\bowtie)$ e-mail: qfliao@whu.edu.cn

J. YANG, Electric Power Research Institute, China Southern

Power Grid, Guangzhou 510080, China

\section{Introduction}

With the development of a long-distance and high-capacity interconnected network, the increasingly enhanced power exchange between regions has brought enormous social and economic benefits [1-3]. However, with the improvement of power network structure and operation mode complexity, the risks of cascading fault and even large power outage increase $[4,5]$. Out-of-step splitting, as an important component of the third defensive line in systems, is of great significance to the prevention of power grid breakdown and large-area power outage. At present, out-of-step splitting device takes catching the electrical center as core criterion means [6-10]. Therefore, studies on the out-of-step splitting criterion have been the hotspot of scholars.

In the out-of-step process of a system, the electrical center, as the lowest voltage point [11], owns the periodic zero passage of circuit active power [12], maximum potential energy, overall infusion of the reactive power at both sides towards the center [13] and $0 \sim 360^{\circ}$ periodical continuous changes of the bus voltage phase angle difference at both sides and other electrical quantity features. Due to the electrical center migrates, the current criterion has the following problems in fitness.

1) When the system oscillation mode is relatively simple, the electrical center does not migrate. $u \cos \varphi$ criterion reflects the lowest voltage point and through the locus of $u \cos \varphi$, it passes through seven regions step by step to catch the electrical center in out-of-step cycle so as to accurately identify the occurrence time of the electrical center [14]. However, it is difficult to determine the specific location of a electrical center. The phase angle criterion is based on the change rules of the phase angle in asynchronism [15]. It can clarify the direction of the electrical center but cannot obtain the detailed location. Apparent impedance locus 
criterion [16] and impedance angle criterion [17] are based on the periodic zero passage principle of out-of-step section active power but the non-electrical center circuit also may appear the zero passage phenomena of active power, which is easy to cause the device misjudgment. Reactive power integral criterion carries out the reactive power integral computation of interconnection lines at both sides in the out-of-step cycle to judge the electrical center [18]. However, its integral origin is difficult to choose and the integration period is not easy to determine. Thus, it lacks practicability. Most of the actual out-of-step splitting devices adopt the compound criterion for better reliability.

2) The electrical center rapidly and dynamically migrates among multiple interfaces due to the oscillation mode of systems and other reasons. Numerous simulations and actual operation accident analyses indicate that after the out-of-step of large-region interconnected network, the adaptability of the above criterion further reduces. For example, $u \cos \varphi$ criterion for lack of the integrity of locus mainly manifests that its locus only passes through few regions and fails to reach the action threshold, leading to the refusing action. Even the compound criterion still fails to solve these inherent problems. The migration of electrical center brings a great challenge to the normal action of out-of-step splitting, which may lead to serious consequences [19]. Therefore, real-time and accurate identification and location of the electrical center and better dealing with the dynamic migration of the electrical center are urgent issues to be solved at present.

The identification and location of the electrical center based on the frequency characteristics is a new try. References [20,21] proposed the frequency characteristics-based location method and relevant criterion of the electrical center. However, these studies are not profound enough, mainly including the problems: 1) The instantaneous frequency of the electric current is not easy to be measured, leading to the difficulty in the actual application; 2) After the out-of-step of power grid, the system frequencies at both sides are not consistent, so that the solution method by phasor diagram should not be adopted. Thus, the analytical method is used; 3 ) The extremum of the voltage frequency curve deduced from the formula is fixed as the frequencies of equivalent potential at both sides of the system, which does not conform to the actual situation. Therefore, the studies of the electrical center based on the frequency characteristics need further explorations.

Given this, this paper firstly makes detailed theoretical derivation and solution on the basis of equivalent twomachine system, obtaining the analytical expression of the instantaneous voltage frequency in the electrical and nonelectrical centers. Also it discusses the distribution of extremum, analyzes the voltage frequency characteristics of electrical center and non-electrical center at different voltage amplitude ratios in case of out-of-step of the power grid and further obtains the voltage frequency change rules in the typical scene of electrical center migration. Finally, out-of-step splitting criterion based on bus voltage frequency is proposed and its accuracy and the effectiveness are verified in CEPRI-36 system and interconnected network example of one actual region.

\section{Out-of-step model}

Due to the out-of-step of power grid, the equivalent power angle of two-generator increases gradually. The outof-step of power grid also can be viewed as the instability of two-generators or instability of successive multiple twogenerators [22, 23], manifested by continuous oscillation of voltages and currents, and inconsistent frequencies at two system sides. Therefore, the variation law of out-of-step oscillation center in a power system can be discussed by establishing an equivalent two-generator model.

Figure 1 shows the equivalent two-generator system model. Two generators at two system sides are represented by the equivalent potential $\dot{E}_{M}$ and $\dot{E}_{N}$. It is assumed that the interconnection wire $A B$ is even and its impedance angle is the same with the system impedance angle. The impedance variation within the variable frequency range is neglected. The impedance from any point $(D)$ to the busbar $(B)$ is $Z_{D B}$.

Actually, since the instantaneous current frequency of power grid after out-of-step is difficult to be gained, this paper focused on the voltage frequency characteristics during the out-of-step of power system. Parameters are expressed by instantaneous values

$\left\{\begin{array}{l}e_{M}=E_{M} \sin \left(\omega_{M} t\right) \\ e_{N}=E_{N} \sin \left(\omega_{N} t\right) \\ u_{D}=U_{D} \sin \left(\omega_{D} t\right)\end{array}\right.$

where $\omega_{M}$ and $\omega_{N}$ are the instantaneous frequencies of equivalent potentials at two system sides; $u_{D}, U_{D}$ and $\omega_{D}$ are instantaneous voltage, voltage effective value and instantaneous frequency of $D$, respectively.

Supposing that $A$ and $B$ are the sending and receiving ends of power system, respectively. At any time, there is

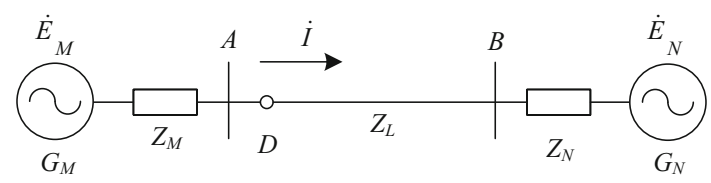

Fig. 1 Equivalent two-generator system 


$$
\begin{aligned}
u_{D}= & e_{N}+c\left(e_{M}-e_{N}\right)=E_{N} \sin \left(\omega_{N} t\right) \\
& +c\left(E_{M} \sin \left(\omega_{M} t\right)-E_{N} \sin \left(\omega_{N} t\right)\right) \\
= & E_{N} \sin \left(\omega_{N} t\right)+c\left(E_{M} \sin \left(\left(\Delta \omega+\omega_{N}\right) t\right)\right. \\
& \left.-E_{N} \sin \left(\omega_{N} t\right)\right) \\
= & E_{N} \sqrt{\left(1-c+c k_{e} \cos (\Delta \omega t)\right)^{2}+\left(c k_{e} \sin (\Delta \omega t)\right)^{2}} \\
& \times \sin \left(\omega_{N} t+\alpha\right)
\end{aligned}
$$

where $c$ is the location coefficient of $D, c=$ $\left(Z_{D B}+Z_{N}\right) / Z_{\Sigma}(0<\mathrm{c}<1) ; \Delta \omega$ is the frequency difference between two system sides $\Delta \omega=\omega_{M}-\omega_{N} ; k_{e}$ is the potential amplitude ratio at two system sides $k_{e}=\frac{E_{M}}{E_{N}}$; $\alpha=\arctan \frac{c k_{e} \sin (\Delta \omega t)}{1-c+c k_{e} \cos (\Delta \omega t)}$.

Therefore, the voltage effective value is

$U_{D}=E_{N} \sqrt{\left(1-c+c k_{e} \cos (\Delta \omega t)\right)^{2}+\left(c k_{e} \sin (\Delta \omega t)\right)^{2}}$

In one out-of-step cycle, the position with minimum $U_{D}$ at any time is called the the oscillation center. When $t=$ $k \pi / \Delta \omega$ ( $k$ is odd), the minimum $U_{D}$ is achieved at $c=$ $1 /\left(1+k_{e}\right)$ where is the electrical center.

Put $\beta=\omega_{N} t+\alpha$ in (1), the instantaneous voltage frequency of $D$ is

$$
\begin{aligned}
\omega_{D}= & \frac{\mathrm{d} \beta}{\mathrm{d} t}=\omega_{N}+\frac{\mathrm{d} \alpha}{\mathrm{d} t}=\omega_{N} \\
& +\frac{c(1-c) k_{e} \cos (\Delta \omega t)+c^{2} k_{e}^{2}}{(1-c)^{2}+2 c(1-c) k_{e} \cos (\Delta \omega t)+c^{2} k_{e}^{2}} \Delta \omega
\end{aligned}
$$

The instantaneous voltage frequency at $D$ changes as time goes on from (4). It is determined by its location, $k_{e}$ and $\Delta \omega$.

For the convenience of theoretical analysis, frequencies at two system sides are believed to be constant within the short out-of-step time. In other word, $\Delta \omega$ is a constant. Researches confirme that such simplification cannot influence the variation law of voltage frequency [24].

Let $\varphi(t)=\frac{c(1-c) k_{e} \cos (\Delta \omega t)+c^{2} k_{e}^{2}}{(1-c)^{2}+2 c(1-c) k_{e} \cos (\Delta \omega t)+c^{2} k_{e}^{2}}$, then the voltage frequency is

$\omega_{D}=\omega_{N}+\varphi(t) \Delta \omega$

Calculate the derivative of $\varphi(t)$,

$\varphi^{\prime}(t)=\frac{c(1-c) k_{e}\left(c^{2} k_{e}^{2}-(1-c)^{2}\right) \sin (\Delta \omega t)}{\left((1-c)^{2}+2 c(1-c) k_{e} \cos (\Delta \omega t)+c^{2} k_{e}^{2}\right)^{2}} \Delta \omega$

Since $\varphi^{\prime}(t), c=1 /\left(1+k_{e}\right)$ or $t=k \pi / \Delta \omega \quad(k$ is an integer). Then, $\omega_{D}$ reaches the minimum or the maximum when
1) $c=1 /\left(1+k_{e}\right)(D$ is the electrical center). Substituting it into (5):

$\omega_{D}=\left(\omega_{M}+\omega_{N}\right) / 2=\omega_{a v}$

where $\omega_{a v}$ is the mean voltage frequency of two system sides.

2) $1 /\left(1+k_{e}\right)<c<1$.

a) $t=k \pi / \Delta \omega$ ( $k$ is even), $\omega_{D}$ reaches the minimum value.

$\omega_{D \min }=\omega_{N}+\frac{1-c+c k_{e}}{(1-c)^{2}+2 c(1-c) k_{e}+c^{2} k_{e}^{2}} c k_{e} \Delta \omega$

b) $t=k \pi / \Delta \omega$ ( $k$ is odd), $\omega_{D}$ reaches the maximum value.

$\omega_{D \max }=\omega_{N}+\frac{c-1+c k_{e}}{(1-c)^{2}-2 c(1-c) k_{e}+c^{2} k_{e}^{2}} c k_{e} \Delta \omega$

3) $0<c<1 /\left(1+k_{e}\right)$

a) $t=k \pi / \Delta \omega$ ( $k$ is even), $\omega_{D}$ reaches the maximum value:

$\omega_{D \max }=\omega_{N}+\frac{1-c+c k_{e}}{(1-c)^{2}+2 c(1-c) k_{e}+c^{2} k_{e}^{2}} c k_{e} \Delta \omega$

b) $t=k \pi / \Delta \omega$ ( $k$ is odd), $\omega_{D}$ reaches the minimum value:

$\omega_{D \min }=\omega_{N}+\frac{c-1+c k_{e}}{(1-c)^{2}-2 c(1-c) k_{e}+c^{2} k_{e}^{2}} c k_{e} \Delta \omega$

According to the above theoretical analysis, the voltage frequency variation of electrical center and $D$ during the out-of-step of system with different $k_{e}$ is shown in Fig. 2.

Voltage frequency characteristics at different positions with different $k_{e}$ are analyzed according to Fig. 2. When $k_{e}=1.0$, the electrical center falls on the center of $A B$. When $k_{e}=1.2$ and $0.8, c$ is 0.4545 and 0.5556 , respectively. This indicates that the electrical center is closer to the side with smaller voltage amplitude. Moreover, the voltage frequency of electrical center is always equal to the mean voltage frequency at two system sides. Voltage frequencies of different positions at the same side of electrical center show similar continuous periodic changes against time and their voltage frequency curves have point of intersection. It is calculated that the mean voltage frequency of different positions at the same side of electrical center in one cycle is equal to the system frequency of this side. Voltage frequencies at two sides of electrical center change oppositely and their curves have no point of intersection. The voltage frequency closer to the electrical center oscillates more violently and has larger amplitude. 


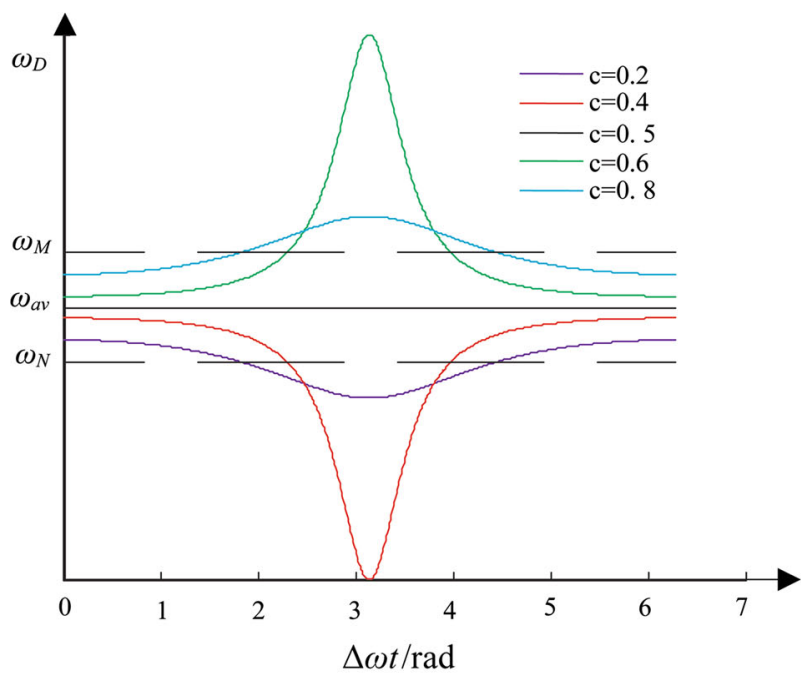

(a) $k_{\mathrm{e}}=1.0$

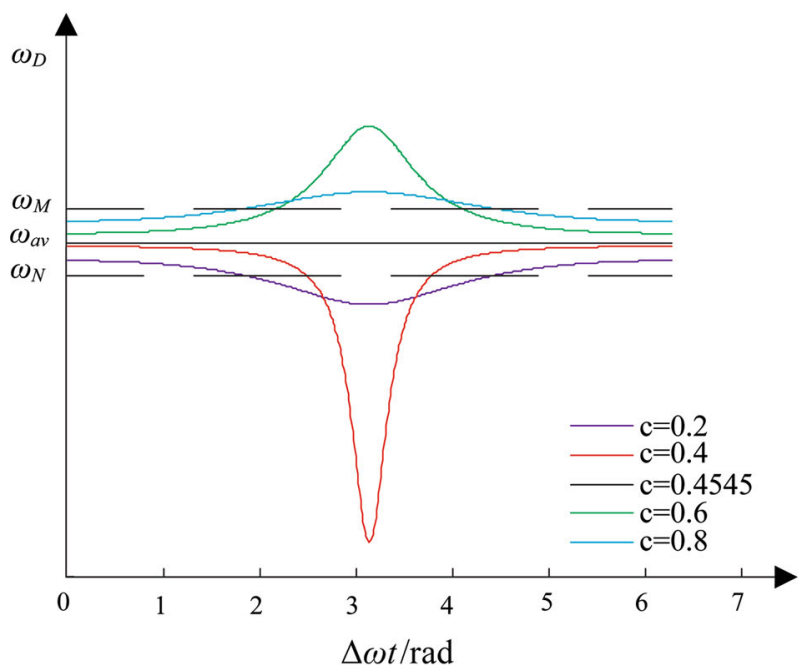

(b) $k_{\mathrm{e}}=1.2$

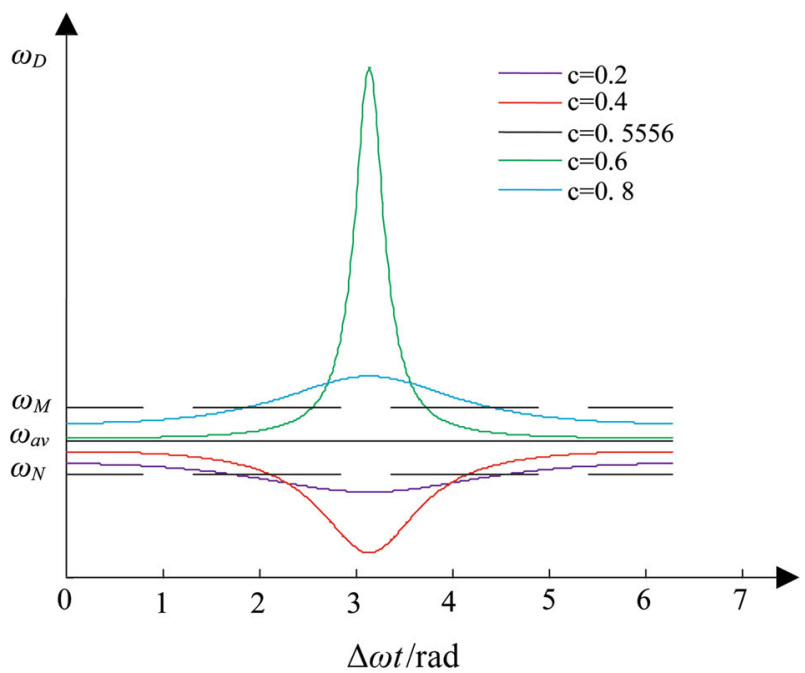

(c) $k_{\mathrm{e}}=0.8$

Fig. 2 Voltage frequency curves at different positions with different $k_{e}$
Otherwise, the voltage frequency oscillates more gently and has smaller amplitude.

\section{Voltage frequency characteristics in migration of electrical center}

In the actual multi-machine system, the electrical center of the grid power sometimes may fall on one section [19], instead of one or two interconnection lines. The sections of two independent sub-networks in the power grid can be defined as the splitting section. Studies show that under the influences on the grid fault mode, oscillation mode, fault clearing time and other factors, the electrical center will dynamically migrate between different sections. Figure 3 shows the typical scene of electrical center migration in the multi-machine system, where red dotted line represents the section of the electrical center, $G_{i}(i=1,2,3)$ represent the equivalent machines and $a \sim f$ represent the buses at both sides of each circuit branch.

After out-of-step, $G_{1}$ is a coherent group, while $G_{2}$ and $G_{3}$ constitute another coherent group. At $t_{0}$, the oscillation mode changes. $G_{1}$ and $G_{3}$ constitute one coherent group and $G_{2}$ becomes a coherent group. The bus voltage frequency changes at both sides of each circuit branch are shown in Fig. 4.

According to the above analysis on the frequency voltage characteristics in the out-of-step oscillation, before $t_{0}$, the electrical center is located in Sect. 1. It is constituted of circuit branches $a b$ and $c d$. At $t_{0}$, the frequency of bus $c$ decreases and reverses the direction to intersect with the frequency curve of bus $d$, presenting similar change rule. At the same time, the frequency of bus $f$ decreases and reverses the direction, which starts to be opposite to the change of the frequency curve of bus $e$. At this time, the electrical center migrates to Sect. 2.

Therefore, despite that there are many complex and changeable reasons for electrical center migration in actual grid fault, the voltage frequency of migration satisfies the following rules all the time, namely, during the migration of the electrical center, the bus frequency at one side of electrical center circuit branch starts to have similar

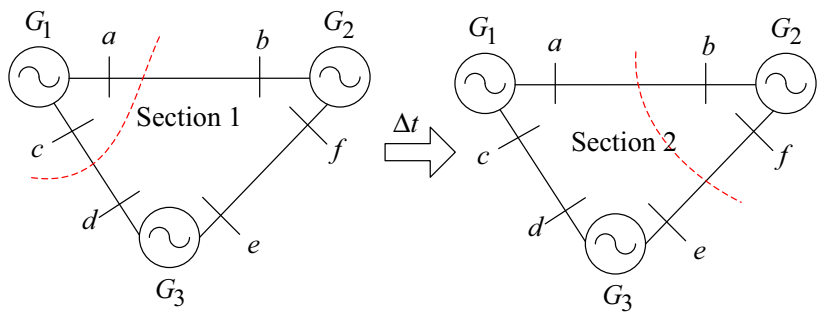

Fig. 3 Typical scene of electrical center migration 


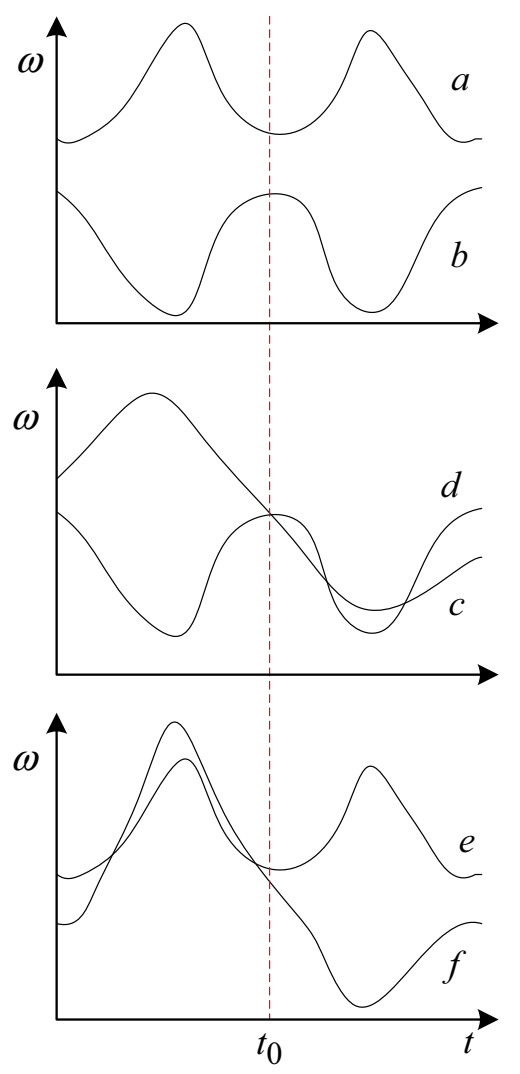

Fig. 4 Bus voltage frequencies at both sides in electrical center migration

changes to the other side. After the migration of the electrical center, the frequency curve at one side of new electrical center circuit branch starts to have an opposite change trend with the other side.

\section{Splitting criterion based on bus voltage frequency}

With account step of $t_{\text {step }}$, the bus voltage frequency difference at both sides of circuit branches $L 1-2$ at $t$ can be defined as $A_{u 12, t}=\omega_{u 1, t}-\omega_{u 2, t}$. The frequency difference increment is defined as $B_{u 12, t}=\left|A_{u 12, t+t_{\text {step }}}\right|-\left|A_{u 12, t}\right|$, and the voltage frequency increment is defined as $C_{u i, t}=\omega_{u i, t+t_{\text {step }}}-\omega_{u i, t}(i=1,2)$.

It can be known from the above analysis that the voltage frequencies at both sides of the electrical center in one outof-step cycle has two features: 1) opposite voltage frequency locus change; 2) the absolute value of frequency difference increases firstly and then decreases, where there is a maximum.

Supposing that the electrical center lies in circuit branches $L 1-2$. The changes of $B_{u 12, t}$ and $C_{u 1, t} C_{u 2, t}$ are shown in Figs. 5 and 6, respectively.

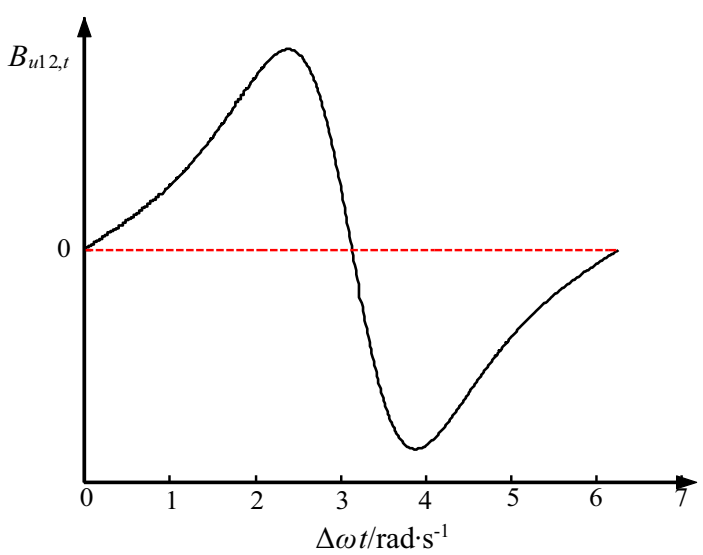

Fig. 5 Bus voltage frequency increments at both sides of L1-2

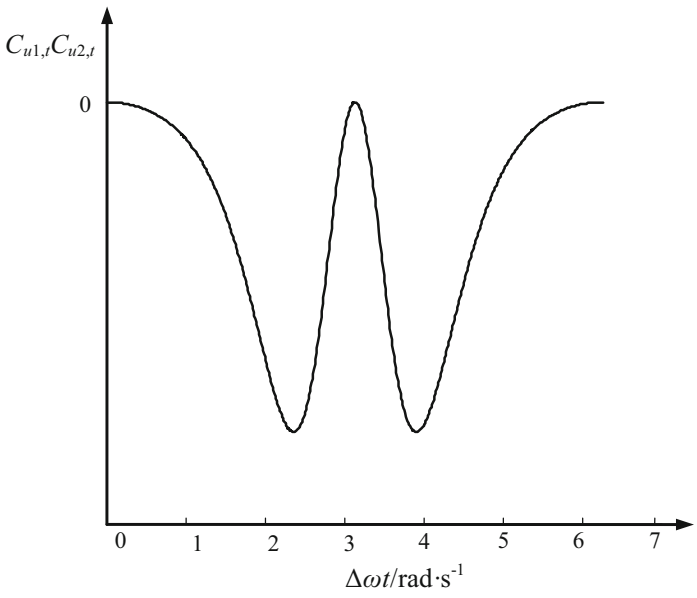

Fig. 6 Bus voltage frequency increments at both sides of L1-2

It can be seen from Fig. 5 that the frequency difference increment $B_{u 12, t}$ in one out-of-step cycle changes from positive to negative and passes zero for once, which corresponds to the maximum of the voltage frequency difference at both sides of buses. It reflects the firstly increasing and then decreasing feature of the voltage frequency difference at both sides of buses. Figure 6 shows that the voltage frequency increment at both sides of buses keeps non-positive, which reflects the opposite voltage frequency locus at both sides of buses in the electrical center. Therefore, only when the above two conditions are satisfied at the same time, the electrical center can be accurately caught. The formulated criterion expression can be expressed as

$B_{u 12, t}>0$

$C_{u 1, t} C_{u 2, t} \leq 0$ 
$B_{u 12, t} B_{u 12, t+t_{\text {step }}}<0$

Once the electrical center migrates, the voltage frequency locus at both sides of buses in the electrical center will become reverse and the expression of the migration criterion is

$A_{u 12, t} A_{u 12, t+t_{\text {step }}}<0$

The bus voltage frequencies at both sides of all circuits are real-time monitored. A mass of simulations of the simplified system of the IEEE standard system model show that when the bus voltage phase angle differences at both sides of the interconnected system has exceeded a given threshold (such as $130^{\circ}$ ) and continues to enlarge, the power system will be out-of-step [25].

The criterion of bus voltage phase angle differences is a traditional method to capture the electrical center. The shortcomings are listed as follows:

1) The bus voltage phase angle differences cannot take the place of power angle differences and the former is just the approximation of the latter. Reference [26] studied the influence factors of the relationship of bus voltage phase angle differences and power angle differences. The influence factors include the middle load between the equivalent two-generator system and the voltage amplitude ratio at two branch sides, etc.

2) Reference [13] pointed out that when the short circuit fault occurs on the branch, the bus voltage phase angle differences at both sides of the branch may also be $180^{\circ}$, resulting in the device misjudgment.

Thus, in order to avoid the defects of phase angle differences, the out-of-step splitting criterion based on bus voltage frequency is proposed with the bus voltage phase angle differences at both sides of the branch $\Delta \theta>130^{\circ}$ as the startup criterion.

The initial value $t_{0}$ of the parameter $t$ in the equation above is defined as the moment when the startup criterion acts. When (12)-(14) are satisfied simultaneously, the electrical center can be determined by locating on circuit branch. Further, the location of the electrical center within half out-of-step cycle can be realized. If the section constituted of the circuit branch of the electrical center is splitting section, the time node can be determined according to the practical situation and then the splitting order is sent and the splitting operation is implemented. If not, continue monitoring until the splitting section occurs. If (15) is satisfied during the monitoring period, it is indicated that the electrical center migrates and it needs locating again. The applicable scope of criterion is that there are two coherent generators when the system is outof-step. Figure 7 shows the flow diagram of bus voltage frequency-based splitting criterion.

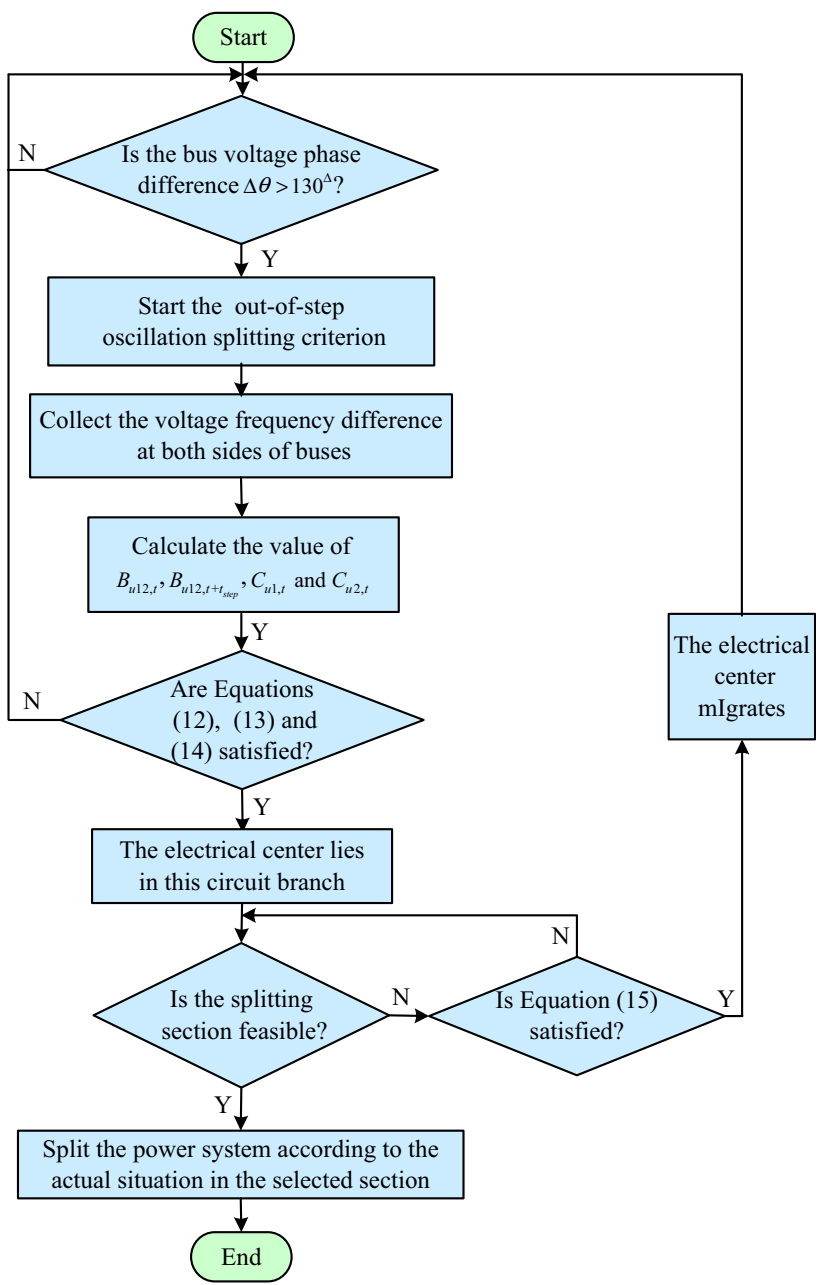

Fig. 7 Flow chart of bus voltage frequency-based splitting criterion

\section{Simulation analysis}

\subsection{CEPRI-36 system example}

Simulation calculation is carried out based on power system analysis software package (PSASP) and the CEPRI36 standard system of China Electric Power Research Institute is adopted. In an operation mode, the output of generator set in the whole network is 26.78 (per unit value, with reference value of $100 \mathrm{MW}$ ), with the load of 25.69 , frequency standard of $50 \mathrm{~Hz}$ and account step $0.01 \mathrm{~s}$.

Three-phase short circuit ground fault occurs at the circuit branch $L 19-30$ at $0 \mathrm{~s}$ and the fault is cleared at $0.2 \mathrm{~s}$. The relative power angle curve of the generator is shown in Fig. 8 (taking $G_{1}$ as reference machine).

Figure 8 shows the relative power angles of generators $G_{2} \sim G_{8}$ and $G_{1}$. Around $1.37 \mathrm{~s}, G_{7}$ and $G_{8}$ start to lose stability compared with the left units. The bus voltage phase angle differences at both sides of all circuit branches are monitored and changes within $0 \sim 360^{\circ}$ according to the 
bus voltage phase angle differences at both sides of the electrical center and are continuous by $180^{\circ}$. It is found that only circuit branch L19-30 and circuit branch L33-34 conform to this feature, respectively, as shown in Fig. 9.

It can be known from Fig. 9 that after the fault is cleared the electrical center lies in circuit branch L19-30 and L3334 and does not migrate in the simulation time. Figure 10 indicates that the voltage frequency locus changes at both sides of circuit branch L19-30 and L33-34 are opposite and the absolute value of the frequency difference increases firstly and then decreases, with maximum, which conforms to the voltage frequency characteristics of the electrical center.

It can be seen from Fig. 9 that at $0.71 \mathrm{~s}$ the relative power angle $\Delta \theta=133^{\circ}>130^{\circ}$. At this time, the criterion launches. According to the criterion proposed in this paper, the bus voltage frequency differences and bus voltage frequency increments at both sides of L19-30 and L33-34 are shown in Table 1.

It can be seen from Table 1 that at $1.44 \mathrm{~s}$ circuit branch L19-30 can satisfy (12) and (13) simultaneously. At this time, the criterion launches. At $1.44 \mathrm{~s}$, it satisfies (12) (14) simultaneously, indicating that the bus frequency change at both sides are opposite and the absolute value of the frequency difference at both sides reaches the maximum at $1.50 \mathrm{~s}$. Therefore, it satisfies frequency criterion and in half out-of-step cycle the electrical center is determined to locate on circuit branch L19-30. Similarly, it can be known that the electrical center also is located on circuit branch L33-34. L19-30 and L33-34 can constitute splitting section, thus spliting the device action and implementing the splitting operation. Any circuit branch is selected and monitor its active power is monitored, as shown in Fig. 11.

Figure 11 reflects that the active power of circuit branch after splitting returns to stable change, proving the effectiveness of the splitting.

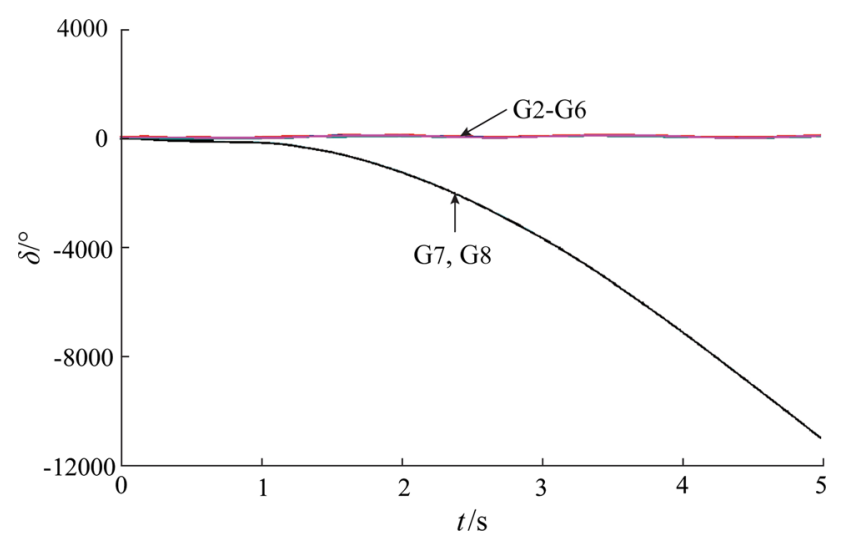

Fig. 8 Relative power angle of CEPRI-36 system generator

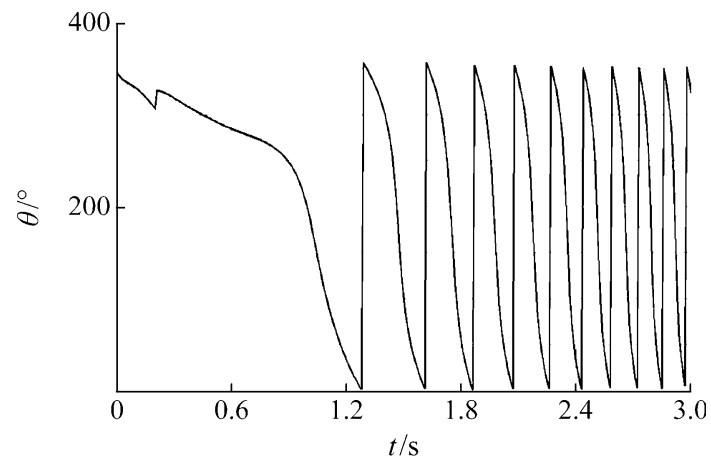

(a) Circuit branches L19-30

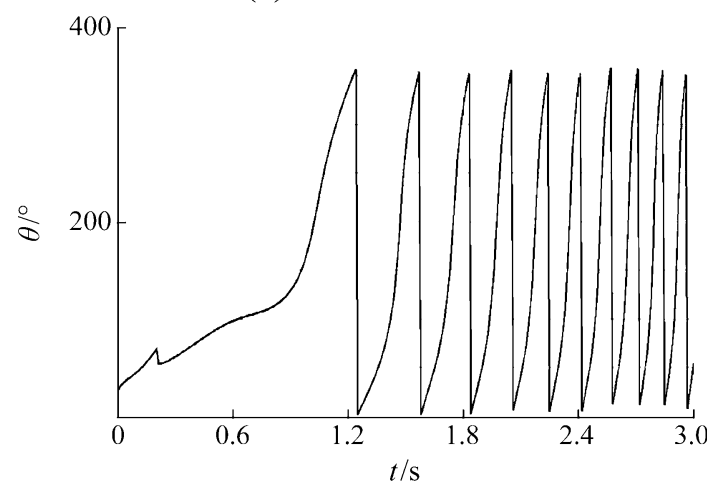

(b) Circuit branches L33-34 4

Fig. 9 Bus voltage phase angle differences

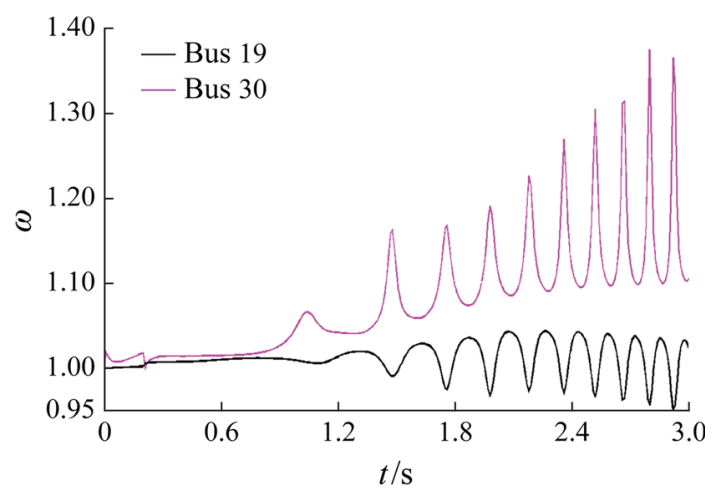

(a) $L 19-30$

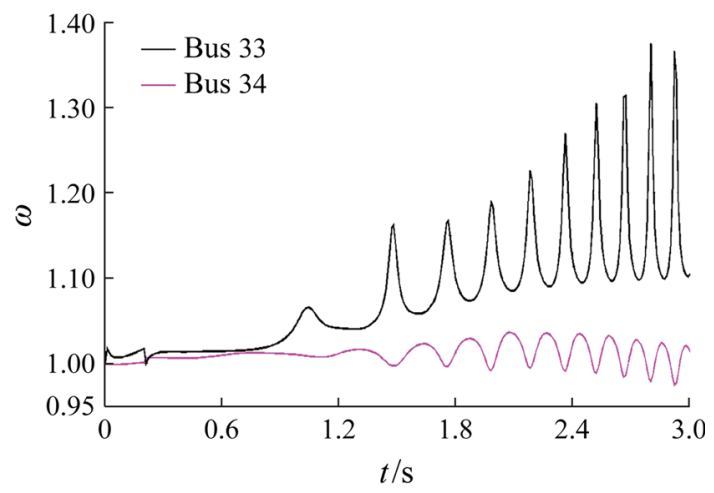

(b) $L 33-34$

Fig. 10 Bus voltage frequencies 
Table 1 Bus voltage frequency differences and bus voltage frequency increments at both sides of $L 19-30$ and $L 33-34$

\begin{tabular}{|c|c|c|c|c|c|}
\hline Circuit branch & $t / s$ & $C_{u 1, t}\left(\times 10^{-3}\right)$ & $C_{u 2, t}\left(\times 10^{-3}\right)$ & $C_{u 1, t} C_{u 2, t}$ & $B_{u 12, t}\left(\times 10^{-3}\right)$ \\
\hline \multirow[t]{7}{*}{ L19-30 } & 1.44 & -0.62 & 1.74 & $<0$ & $2.36>0$ \\
\hline & 1.45 & -1.29 & 2.55 & $<0$ & $3.84>0$ \\
\hline & 1.46 & -2.25 & 4.20 & $<0$ & $6.45>0$ \\
\hline & 1.47 & -3.30 & 8.47 & $<0$ & $11.77>0$ \\
\hline & 1.48 & -3.89 & 19.49 & $<0$ & $23.38>0$ \\
\hline & 1.49 & -2.74 & 26.06 & $<0$ & $28.80>0$ \\
\hline & 1.50 & 0.73 & -22.07 & $<0$ & $-28.80<0$ \\
\hline \multirow[t]{7}{*}{ L33-34 } & 1.44 & 1.74 & -0.70 & $<0$ & $2.44>0$ \\
\hline & 1.45 & 2.56 & -1.18 & $<0$ & $3.74>0$ \\
\hline & 1.46 & 4.20 & -1.73 & $<0$ & $5.93>0$ \\
\hline & 1.47 & 8.48 & -2.14 & $<0$ & $10.62>0$ \\
\hline & 1.48 & 19.53 & -2.09 & $<0$ & $21.62>0$ \\
\hline & 1.49 & 26.07 & -1.25 & $<0$ & $27.32>0$ \\
\hline & 1.50 & -22.16 & 0.34 & $<0$ & $-22.50<0$ \\
\hline
\end{tabular}

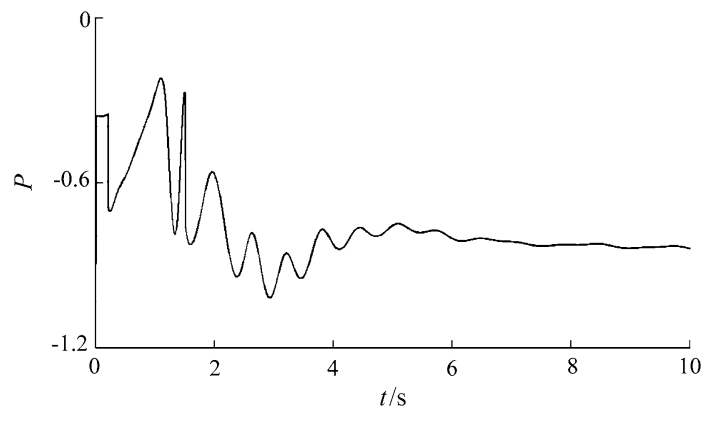

Fig. 11 Active power of circuit branch after splitting

\subsection{Actual power grid example}

Figure 12 shows the $500 \mathrm{kV}$ grid structure diagram of the interconnected network in one actual region. This power grid contains six regional power grids in total. Among them, HB, HUN, HN and JX regions are named as major networks. Between SC region, CQ region and $\mathrm{HB}$ region are two-channel constituted of two loops. In these three regions, there are 148, 20 and 87 generators, respectively. In one operation mode, SC region transmits $4578 \mathrm{MW}$ power to CQ region and CQ region transmits 2044 MW power to HB region.

At $0 \mathrm{~s}$, three-phase short circuit earth fault occurs at circuit branch $L$ (JS-SZ) in SC region and the fault is cleared at $0.19 \mathrm{~s}$. The relative power angle curve of the generator is shown in Fig. 13 (taking DJ unit in HB region as reference machine).

According to Fig. 13, power angles of the major units in $\mathrm{SC}$ region and $\mathrm{CQ}$ region and the major network region at $1.21 \mathrm{~s}$ lay out and lose stability. However, voltage phase angle differences at both sides of interconnection line

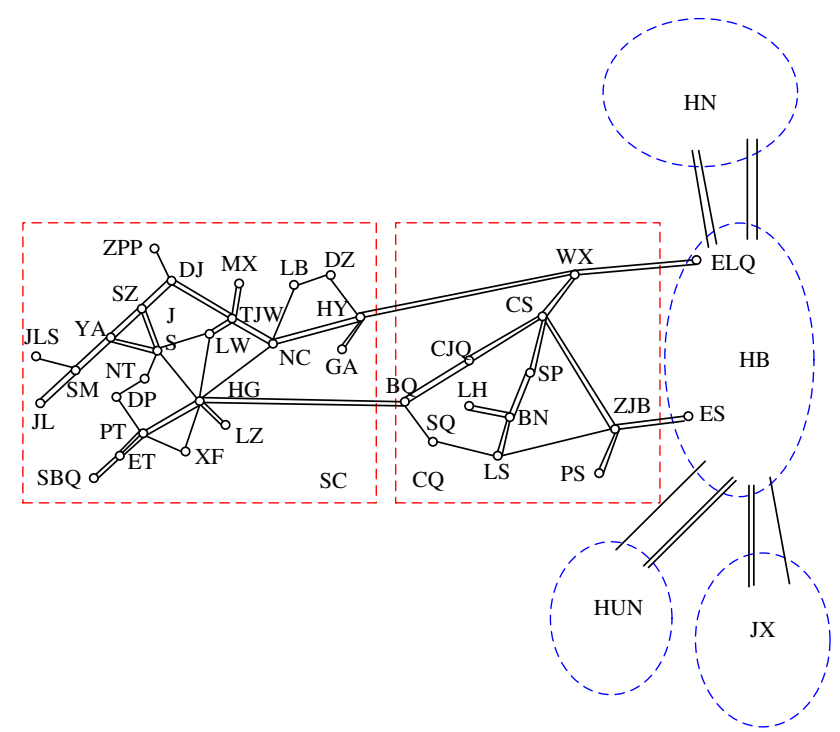

Fig. $12500 \mathrm{kV}$ grid structure of interconnected network in an actual region

$L$ (ZJB-ES) and $L(\mathrm{WX}-\mathrm{LQ})$ in CQ region and $\mathrm{HB}$ region are shown in Fig. 14.

It can be seen from Fig. 14 that after the asynchronism of the system, the electrical center rapidly migrates on circuit branch $L(\mathrm{ZJB}-\mathrm{LS})$ and $L(\mathrm{ZJB}-\mathrm{ES})$, while the electrical center occurs on circuit branch $L(\mathrm{WX}-\mathrm{LQ})$ until 2.55. The bus voltage frequencies at both sides of the above circuit branches are shown in Fig. 15.

In Fig. 15a, the changing trend of bus voltage frequencies at both sides of circuit branches reflects the constant and rapid migration of electrical center on circuit branch $L$ (ZJB-CS) and L(ZJB-ES), which conforms to Fig. 14. It 


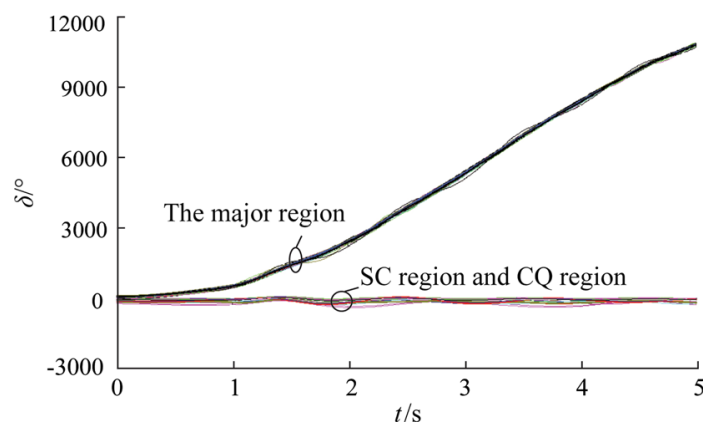

Fig. 13 Relative power angle curves of generator in an interconnected network

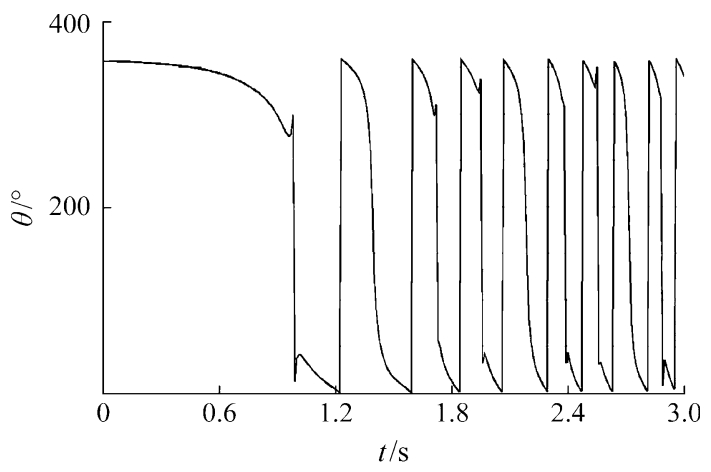

(a) $L$ (ZJB-LS)

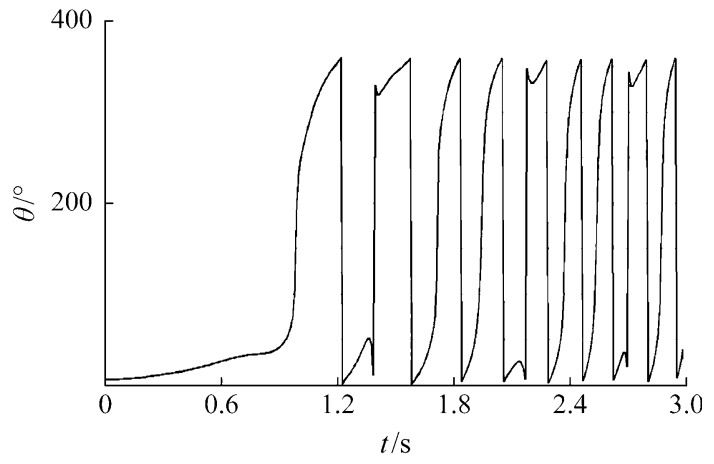

(b) $L$ (ZJB-ES)

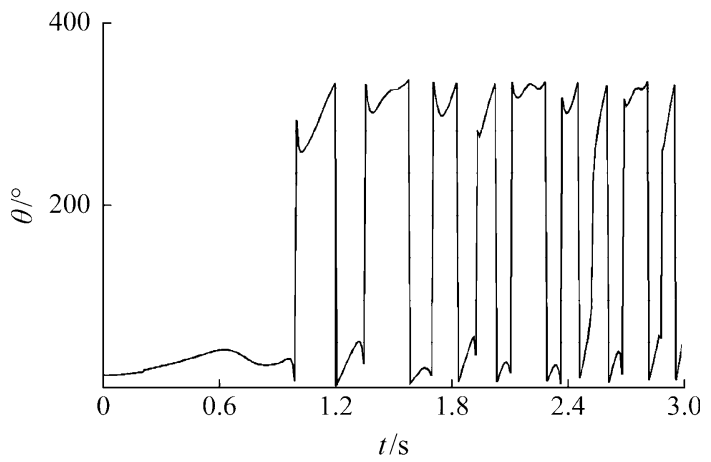

(c) $L(\mathrm{WX}-\mathrm{LQ})$

Fig. 14 Bus voltage phase angle differences at both sides of circuit branches can be seen from Fig. 14 that at $1.05 \mathrm{~s}$ the relative power angle $\Delta \theta=136^{\circ}>130^{\circ}$. At this time, the criterion launches. Using the criterion proposed in this paper, bus voltage frequency differences and bus voltage frequency increments at both sides of the above circuit branches on some time nodes are provided to illustrate the location of the electrical center due to the limited space, as shown in Table 2 .

According to Table 2, at $1.95 \mathrm{~s}$ circuit branch $L(\mathrm{ZJB}-$ ES) satisfies (12)-(14) simultaneously while circuit branch $L$ (ZJB-LS) fails to satisfy (14). Therefore, the electrical center lies in circuit branch $L(Z J B-E S)$ while the circuit branches of the electrical center do not constitute splitting section. Therefore, it needs further monitoring. At $2.15 \mathrm{~s}$, circuit branch $L(Z J B-E S)$ satisfies (15) and the electrical center migrates on circuit branch $L(Z J B-E S)$. Thus, the electrical center needs relocating. At $2.18 \mathrm{~s}$, circuit branch $L$ (ZJB-LS) satisfies (12)-(14) simultaneously and the electrical center migrates from circuit branch $L(Z J B-E S)$ to circuit branch $L(\mathrm{ZJB}-\mathrm{LS})$. However, the circuit branches of the electrical center do not constitute splitting section. At $2.38 \mathrm{~s}$, circuit branch $L$ (ZJB-LS) satisfies (15) and the electrical center migrates on circuit branch L(ZJB-LS). Thus, the electrical center needs relocating. Until $2.55 \mathrm{~s}$, the electrical center lies in circuit branch $L(\mathrm{ZJB}-\mathrm{LS})$ and

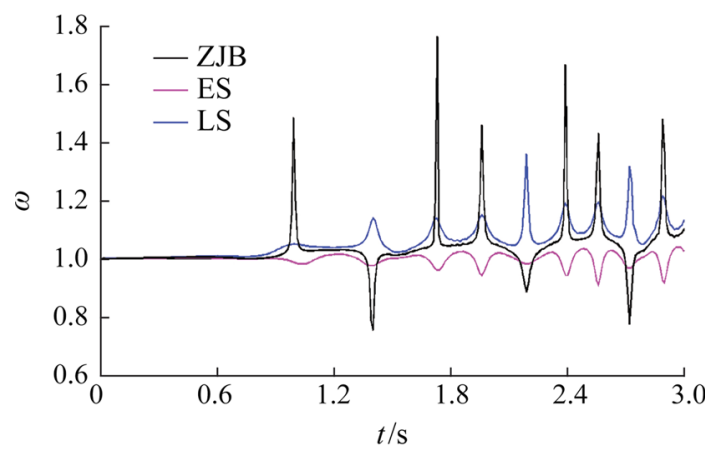

(a) $L$ (ZJB-LS) and $L$ (ZJB-ES)

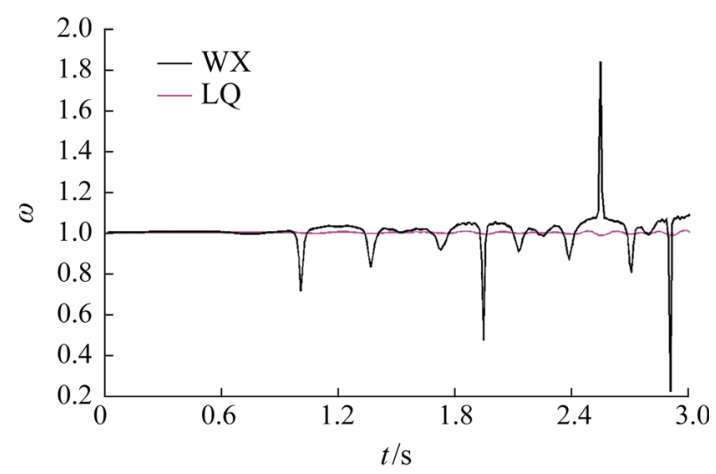

(b) $L$ (WX-LQ)

Fig. 15 Bus voltage frequencies at both sides of circuit branches 


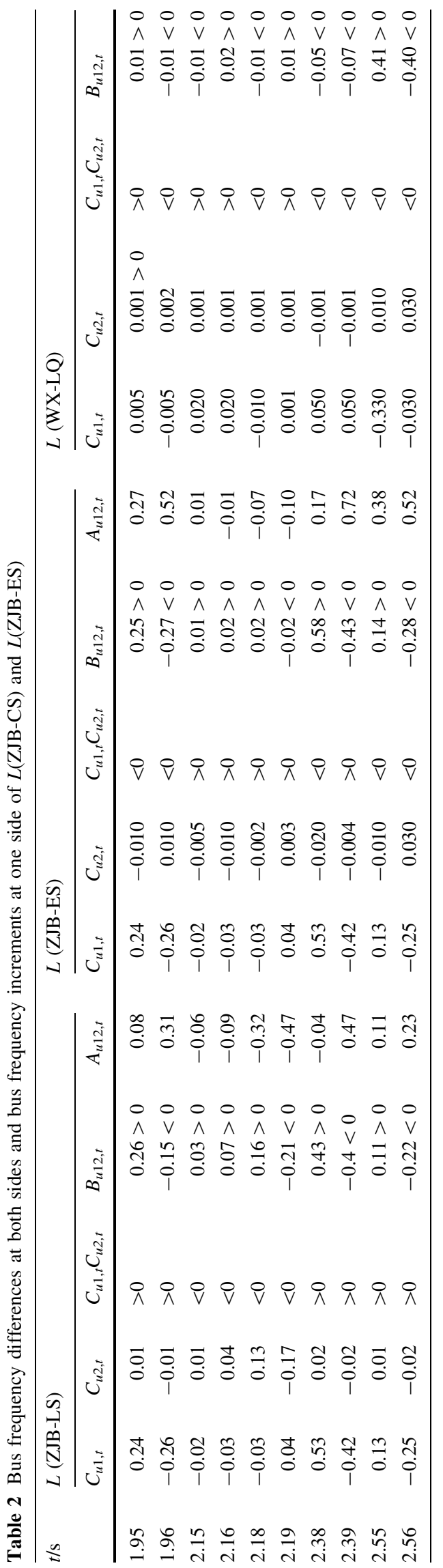

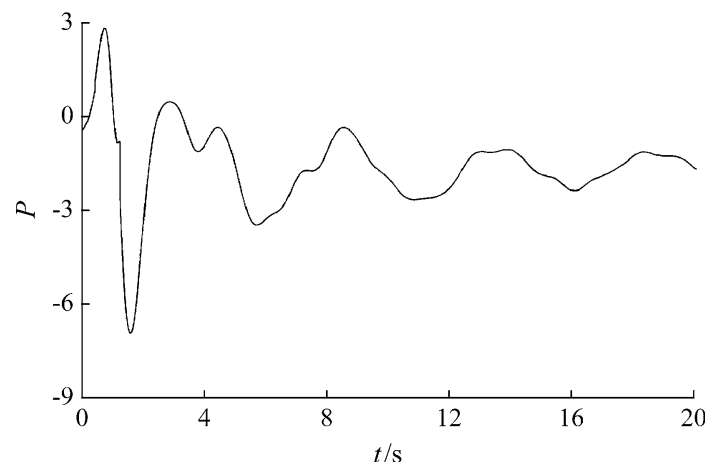

Fig. 16 Active power curve of major network after splitting

circuit branch $L(\mathrm{WX}-\mathrm{LQ})$ simultaneously and the circuit branches of the electrical center can constitute splitting section. According to the actual situation, the time nodes are determined. Issue the splitting order and implement the splitting operation. Figure 16 shows that active power curve of one circuit branch of the major network after splitting, indicating the effectiveness of splitting.

\section{Conclusions}

This paper analyzes the frequency characteristics of the electrical and non-electrical centers at different voltage amplitude ratios in case of the out-of-step of power grid and further obtains the voltage frequency change rules of the migration of electrical center in the typical scene. On this basis, the out-of-step splitting criterion based on bus voltage frequency is proposed. This paper draws the following conclusions:

1) In the out-of-step of the power grid, the instantaneous voltage frequencies of the electrical and non-electrical centers are relevant to the location, the frequency difference of the equivalent potentials at both sides and amplitude ratio. The voltage frequency locus changes at both sides of the electrical center are opposite. In one out-ofstep cycle, the absolute value of frequency difference increases firstly and then decreases, where there is a maximum. The voltage frequency loci at the same side are similar and the curves intersect.

2) In the migration scene of the electrical center, the bus voltage frequency differences of the electrical center at both sides pass zero and turns reverse, satisfying the rule. During the migration of the electrical center, the bus frequency at one side of the electrical center circuit branch starts to have similar changes to the other side. After the migration of the electrical center, the frequency curve at one side of the new electrical center circuit branch starts to have an opposite change with the other side. 
3) The bus voltage frequency-based out-of-step oscillation splitting criterion proposed in this paper is easy to be realized and the criterion can adapt to the migration of the electrical center and be free from the limits of the power network structure and operation mode. In half out-of-step cycle, the location of the electrical center can be realized and simulation calculation is implemented in two typical scenes, non-migration and rapidly dynamic migration of the electrical center. Results verify the accuracy and the effectiveness of the criterion.

Acknowledgments This work was supported by State Grid Corporation of China,Major Projects on Planning and Operation Control of Large Scale Grid (No. SGCC-MPLG029-2012) and China Postdoctoral Science Foundation (No. 2014M552080).

Open Access This article is distributed under the terms of the Creative Commons Attribution 4.0 International License (http:// creativecommons.org/licenses/by/4.0/), which permits unrestricted use, distribution, and reproduction in any medium, provided you give appropriate credit to the original author(s) and the source, provide a link to the Creative Commons license, and indicate if changes were made.

\section{References}

[1] Ren XC, Li W, Xue YS et al (2013) Unstable modes evolution and its influencing factors analysis in interconnected power grids. Autom Electr Power Syst 37(21):9-16. doi:10.7500/ AEPS20130901001 (in Chinese)

[2] Wang YT, Tang Y, Ding LJ et al (2013) Research and development of new wide area out-of-step control technology for power systems. Power Syst Technol 37(7):1827-1833 (in Chinese)

[3] Li Q, Lin T, Gao YX et al (2010) Simulation of transients in interconnection test of regional power grids. Power Syst Technol 34(3):85-90 (in Chinese)

[4] Tang Y, Bu GQ, Yi J (2012) Analysis and lessons of the blackout in Indian power grid on July 30 and 31, 2012. P CSEE 32(25):167-174 (in Chinese)

[5] Fang YJ (2013) Application of emergency control to reduce risk of system collapse triggered by power transmission interface tripping: thinking on the India power blackouts. Autom Electr Power Syst 37(4):1-6 (in Chinese)

[6] Senroy N, Heydt GT (2006) A conceptual framework for the controlled islanding of interconnected power systems. IEEE Trans Power Syst 21(2):1005-1006

[7] Shrestha B, Gokaraju R, Sachdev M (2013) Out-of-step protection using state-plane trajectories analysis. IEEE Trans Power Deliv 28(2): 1083-1093

[8] Danku MA, Baldwin TL (2009) Fast prediction of the powerswing curve across transmission lines during wide area disturbances. In: Proceedings of the 2009 power systems conference (PSC'09), Clemson, SC, USA 10-13 Mar 2009, 5 pp

[9] Dubey R, Samantaray SR, Babu BC et al (2011) A novel out-ofstep detection algorithm using angle of power signal. In: Proceedings of the 2011 international conference on power and energy systems (ICPS'11), Chennai, India, 22-24 Dec 2011, $6 \mathrm{pp}$

[10] Soman SA, Nguyen TB, Pai MA et al (2004) Analysis of angle stability problems: a transmission protection systems perspective. IEEE Trans Power Deliv 19(3):1024-1033
[11] Gao P, Wang JQ, Zhou WP et al (2005) A study on oscillation center. P CSU-EPSA 17(2):48-53 (in Chinese)

[12] Gao P, Wang JQ, Gan DQ et al (2005) Review on power system out-of-step separation. Autom Electr Power Syst 20(19):90-96 (in Chinese)

[13] Zhang BH, Zhang YG, Liu HT (2001) Study on principle of power system separation device based on local electrical parameter. P CSEE 21(12):67-72 (in Chinese)

[14] Zong HL, Ren ZY, Zheng YP et al (2003) A out-of-step splitting device based on the changing track of the voltage of oscillation center. Autom Electr Power Syst 27(19):83-85 (in Chinese)

[15] Dong XJ, Zhao J, Ling C et al (2010) Research on out-of-step oscillation criterion mechanism based on principle of phase angle. Power Syst Protect Contr 38(7):1-6 (in Chinese)

[16] Zhu SS (1995) Principles and techniques of protective relaying for HV grid. China Electric Power Press, Beijing, pp 268-270 (in Chinese)

[17] Gao P, Wang JQ, Zhou WP (2004) Improvement of the apparent impedance angle separation criterion. Autom Electr Power Syst 28(24):36-41 (in Chinese)

[18] Gao P, Wang JQ, Zhou WP et al (2005) Theoretical study on capturing the separation interface of out-of-step based on reactive power. Autom Electr Power Syst 29(5):15-20 (in Chinese)

[19] Fang YJ (2007) Adaptive islanding control of power systems. Autom Electr Power Syst 31(20):41-44, 48 (in Chinese)

[20] Wu LL, Huang SF (2013) Out-of-step oscillation criterion based on frequency characteristics. Electr Power Autom Equip 33(1):18-22 (in Chinese)

[21] Huang SF, Wu LL (2012) Power system oscillation identification based on frequency differences between electrical data. Autom Electr Power Syst 36(23):18-22 (in Chinese)

[22] Liu YQ, Liu YT (2008) An islanding cutset searching approach based on dispatching area. Autom Electr Power Syst 32(11):20-24 (in Chinese)

[23] Xue YS (1998) Quantitative study of general motion stability. Autom Electr Power Syst 22(1):12-17 (in Chinese)

[24] Lei XP (2008) The research and analysis on out-of-step splitting of large-scale interconnected power system. Master Thesis, Xi' an University of Technology, Xi'an, China (in Chinese)

[25] Li L (2009) Studies on out-of-step splitting criterion and control scheme based on wide area measurements. PhD Thesis, Shandong University, Jinan, China (in Chinese)

[26] Zhou LS, Xia CJ, Peng B et al (2001) Study on predictive system separation controls based on PMU. Relay 29(3):9-13 (in Chinese)

Fei TANG received the B.S. degree in Department of Electronics and Information Engineering from Huazhong University of Science and Technology, Wuhan, China, in 2005; the M.S. degree in School of Pattern Recognition and Intelligent System from Huazhong University of Technology and Science, Wuhan, China, in 2007; the Ph.D. degree in School of Electrical Engineering from Wuhan University, Wuhan, China, in 2013. He joined Wuhan University as a post doctor in 2013. His research interests include power system stability and control, and artificial intelligent algorithms application in smart grid.

Jian YANG received the B.S. degree in College of Energy and Electrical Engineering from Hohai University, Nanjing, China, in 2012 and M.S. degree in School of Electrical Engineering from Wuhan University, Wuhan, China, in 2015. Currently, he is a member of Electric Power Research Institute, China Southern Power Grid, Guangzhou, China. His research interests include power system stability and control. 
Qingfen LIAO received the B.S. degree and Ph.D. degree in School of Electrical Engineering from Wuhan University, Wuhan, China, in 2002 and 2007, respectively. Currently, she is an associate professor in School of Electrical Engineering at Wuhan University, Wuhan, China. Her interests are electrical towage and control system.

Yifei WANG received the B.S. degree in School of Electrical Engineering from Wuhan University, Wuhan, China, in 2012. She is currently pursuing the master degree in School of Electrical Engineering at Wuhan university, Wuhan. She is currently a membership of Prof. Dichen LIU's group. Her interests are in the areas of power system dynamic and control.
Jun JIA received the B.S. degree in School of Electrical Engineering from Hefei University of Technology, Hefei, China, in 2013. As a group member of Prof. Dichen LIU, he is currently pursuing the master degree in School of Electrical Engineering at Wuhan University, Wuhan. His research interests include power system stability and control. 